\title{
Severe eosinophilic asthma: a roadmap to consensus
}

\author{
Roland Buhl ${ }^{1}$, Marc Humbert ${ }^{2}$, Leif Bjermer ${ }^{3}$, Pascal Chanez ${ }^{4}$, \\ Liam G. Heaney ${ }^{5}$, Ian Pavord ${ }^{6}$, Santiago Quirce 7 , Johann C. Virchow ${ }^{8}$, \\ Stephen Holgate ${ }^{9}$ and the expert group of the European Consensus \\ Meeting for Severe Eosinophilic Asthma ${ }^{10}$
}

Affiliations: ${ }^{1}$ Dept of Pulmonary Medicine, Mainz University Hospital, Mainz, Germany. ${ }^{2}$ Dept of Respiratory Medicine, Université Paris-Sud, Paris, France. ${ }^{3}$ Dept of Respiratory Medicine and Allergology, Skåne University Hospital, Lund, Sweden. ${ }^{4}$ Dept of Respiratory Medicine, Aix-Marseille University, Marseille, France. ${ }^{5}$ Dept of Respiratory Medicine, Queen's University Belfast, Belfast, UK. ${ }^{6}$ Nuffield Dept of Medicine, University of Oxford, Oxford, UK. ${ }^{7}$ Dept of Allergy Medicine, La Paz University Hospital, Madrid, Spain. ${ }^{8}$ Dept of Pulmonary Medicine, University of Rostock, Rostock, Germany. ${ }^{9}$ Dept of Clinical and Experimental Sciences, University of Southampton, Southampton, UK. ${ }^{10}$ Details of the contributing members of the expert group of the European Consensus Meeting for Severe Eosinophilic Asthma and their affiliations can be found in the Acknowledgements section.

Correspondence: S. Holgate, Southampton General Hospital (Mail Point 810, Level F, South Block), Tremona Road, Southampton, S016 6YD, UK. E-mail: S.Holgatedsoton.ac.uk

@ERSpublications

A task force has identified key consensus issues for defining, diagnosing and treating severe eosinophilic asthma http://ow.ly/zrvO30axYWx

Cite this article as: Buhl R, Humbert M, Bjermer L, et al. Severe eosinophilic asthma: a roadmap to consensus. Eur Respir J 2017; 49: 1700634 [https://doi.org/10.1183/13993003.00634-2017].

\section{Introduction}

Asthma is widely accepted as a complex heterogeneous condition with diverse pathophysiological mechanisms, clinical presentations, comorbidities, physiological characteristics, pathology and outcomes that is typically best managed by a multidisciplinary team [1-4]. Severe asthma is recognised as a major unmet need with a high personal and social impact, as well as a high burden on healthcare resources [4]. As a consequence of advances in the development of precision medicines for patients with severe asthma, the need to identify asthma subtypes by phenotype based on clinical, functional or inflammatory parameters is becoming a mandatory part of management [4-6].

Severe eosinophilic asthma (SEA), also referred to as severe asthma with eosinophilia, is yet to be clearly defined and warrants further discussion. It is a well-recognised but still imprecisely characterised sub-classification of severe asthma, driven by a distinct pathophysiological process involving the abnormal production of type 2 cytokines from T-helper 2 and innate lymphoid cells [3, 4, 7]. Sputum eosinophilia is found in slightly under half of all patients with asthma and both blood and sputum eosinophilia are associated with more severe disease, worse control, and worse prognosis [7]. The consequences of this persistent airway inflammation include air trapping, worsening of symptoms, frequent exacerbations and impact on quality of life [7].

Received: March 272017 | Accepted: March 272017

Support statement: TEVA Pharmaceuticals Europe B.V. provided financial and logistical support for the European Consensus Meeting for Severe Eosinophilic Asthma (November 24, 2016; Amsterdam, The Netherlands) from which discussions this article is derived. In addition, TEVA provided administrative and medical writing support to the authors to prepare this manuscript through their appointed agency (Sciterion London Ltd, London, UK). Funding information for this article has been deposited with the Crossref Funder Registry

Conflict of interest: Disclosures can be found alongside this article at erj.ersjournals.com

Copyright @ERS 2017 
Following recent advances in the mechanistic understanding of eosinophilic disease, which has allowed the introduction of a number of targeted biologic add-on treatments for SEA (licensed and in development), there is a need for guidance aimed at the treating clinician to both define SEA and to indicate by whom, when and how patients should be managed. Guidelines and clinical recommendations have yet to be updated to reflect the latest evidence on these therapies and their potential benefits for patients with SEA. As such, a robust evidence-based consensus on defining SEA in adults, as well as diagnosis, treatment, long-term management and follow-up, is now necessary. In addition, it may eventually be possible to address management of other groups with SEA, such as adolescents, children and the elderly, where there is currently less clinical experience and less trial data [8-15].

\section{Towards a consensus on SEA}

In November 2016, an expert task force of clinicians with an interest in and experience of SEA met to identify the key issues and to set out a roadmap for an independent evidence-based consensus statement intended to be of practical help to colleagues in the respiratory clinic. Figure 1 describes the development process for the meeting and this roadmap editorial.

\section{Diagnosis: what does a patient with SEA look like?}

Early identification of patients with SEA in clinical practice is important; however, identifying these patients in day-to-day practice is not always straightforward [5, 16, 17]. A key initial step, as described by the European Respiratory Society (ERS)/American Thoracic Society (ATS) severe asthma guidelines, is to confirm the diagnosis of asthma and address comorbidities. Secondly, adherence to therapy and inhaler technique must be assessed before a diagnosis of severe uncontrolled asthma refractory to treatment can be confirmed [4]. This phenotype has been variously described in the literature and cluster analysis suggests a phenotype of patients with late-onset, eosinophilic, inflammation-predominant asthma [18].

Adult-onset asthma patients with a high blood eosinophil count $\left(\geqslant 0.3 \times 10^{9}\right.$ per $\left.\mathrm{L}\right)$ have been found to have a distinct phenotype of severe asthma with frequent exacerbations and poor prognosis [19]. Persistent airflow limitation and distal inflammation with air trapping are common in these patients, as is upper airway pathology such as chronic rhinosinusitis with nasal polyposis [17]. The characteristics described in these and other studies are not ubiquitous in all patients (some are found in almost all patients and some in a minority). As such, classification using a set of major and minor diagnostic criteria may help to identify this pattern (table 1).

Asthma patients with atopy will not necessarily have an allergic aetiology to their asthma [20]. Furthermore, it remains to be validated in atopic patients with blood eosinophilia, in the context of a thorough patient history, whether or not age at asthma onset, association between allergen exposure and asthma symptoms/ exacerbations, and presence of fixed airflow obstruction and/or upper airway comorbidities can help to differentiate between severe allergic and severe eosinophilic asthma.

Late 2016
Initial discussions and formation of the initial group
Late 2016
Initial review of the literature conducted
Nov 2016
Kick-off meeting
Diagnosis: what does a patient with SEA look like?
Treatment: how do we treat patients with SEA?
Monitoring and long-term follow-up: what monitoring is
necessary, how do we follow up and how do we determine
who has responded?
Late 2017
ERS research seminar
Publication of a position paper
Proposal for an ERS task force
Proposal for a session at a future ERS Congress
2018
ERS consensus statement or guideline update for management
of adults/adolescents with severe refractory asthma

FIGURE 1 The biologics consensus roadmap ERS consensus statement or guideline update for management
of adults/adolescents with severe refractory asthma 
TABLE 1 Possible diagnostic scheme for severe eosinophilic asthma (SEA)

Major criteria

Diagnosis of severe asthma

Evidence of high-load eosinophilic disease (persistent blood or sputum eosinophilia detected on $\geqslant 2$ occasions)

Frequent exacerbations ( $\geqslant 2$ per year)

Dependence (continuous or intermittent) on oral corticosteroids to achieve asthma control

\section{Minor criteria}

\author{
Late onset of disease \\ Upper airway disease li.e. chronic rhinosinusitis, often \\ with nasal polyposis) \\ Role of other biomarkers (e.g. Feno, periostin and DPP-4) \\ Fixed airflow obstruction \\ Air trapping/presence of mucus plugs
}

DPP-4: dipeptidyl peptidase-4; FenO: exhaled nitric oxide fraction.

Biomarkers have been widely studied in SEA and, in clinical practice, blood eosinophil testing is readily available. There is a consistent relationship between blood and sputum eosinophilia [21]. Evidence exists that blood eosinophilia is a superior biomarker and that those patients with raised eosinophil counts have a higher frequency of severe exacerbation, poorer disease control and show a greater response to anti-eosinophilic therapies $[10,19,22]$. There is evidence to support the predictive value of eosinophils in response to these therapies, with a range of responses at different blood eosinophil levels, and patients with higher blood eosinophil levels $\left(e . g . \geqslant 300\right.$ cells $\left.\mu \mathrm{L}^{-1}\right)$ tending to have a better response to treatment $[14,22]$. Overall, there remains a degree of debate about the optimal use and interpretation of biomarkers for SEA in clinical practice, and this revolves around two main concerns in particular. 1. The magnitude and persistence of eosinophilic disease (the level of eosinophilia which may indicate SEA in different patients, the number of test results needed to confirm SEA, and the management of the complications arising from oral corticosteroid use masking systemic eosinophilia and delaying diagnosis). 2. The role of other biomarkers such as exhaled nitric oxide fraction ( $\mathrm{FeNO}$ ), serum periostin and dipeptidyl peptidase-4 (DPP-4).

A key aim is to develop a clinically useful aid to the identification and diagnosis of patients with severe asthma where eosinophils probably play an important role in the pathophysiology of disease. Based on a system of major and minor criteria to accurately phenotype these patients, each subject will need to meet a specific number or combination of criteria in order to reach a diagnosis of SEA. Robust validation of any tools will be required to ensure applicability.

\section{Treatment: how do we treat patients with SEA?}

Patients with severe asthma who have an eosinophilic-driven phenotype may benefit from newly developed precision medicines. One group clearly eligible for treatment with biologics is those patients with severe asthma adherent to high-dose inhaled corticosteroids who nevertheless have frequent exacerbations with or without symptoms, and who have persistently elevated levels of circulating blood eosinophils.

There are number of targeted therapies, both licensed and in development, that are appropriate for consideration in this patient population. Three biologic therapies are now licensed for severe asthma: omalizumab (anti-immunoglobulin E (IgE)), mepolizumab (anti-interleukin-5 (IL-5)) and reslizumab (anti-IL-5). Four more are currently in the development process: benralizumab (anti-IL-5R $\alpha$ ) has recently reported successful phase 3 results; tralokinumab (anti-IL-13) and dupilumab (anti-IL-4R $\alpha$ ) are in phase 3 trials; and pitrakinra (IL-4 and IL-13 antagonist) has completed phase 2 trials (all as of April 2017). Guidance is needed to understand how best to use these different treatments in practice, tailoring treatment choice to the clinical picture and selecting patients likely to benefit.

Anti-IgE therapy may reduce airway and blood eosinophils and result in less frequent exacerbations for patients with severe persistent allergic asthma. However, the drug is approved only for patients with severe allergic asthma due to perennial allergens and not all patients with uncontrolled asthma respond to anti-IgE therapy. It is not yet clear if persistent eosinophilic inflammation predicts a response to omalizumab, in particular in non-atopic asthma [5], and there is no real predictive factor available to manage this treatment [23]. However, methods such as basophil allergen threshold sensitivity may be effective at monitoring and evaluating response in different populations of patients taking omalizumab. This tool has been studied in patients with severe nut allergy to evaluate treatment efficacy, and has been explored as a biomarker in a small cohort of children with different manifestations of persistent allergic asthma [24, 25]. Interleukin-5 (IL-5) plays a critical role in eosinophil differentiation, maturation, recruitment, survival and activation in tissues. Anti-IL-5 therapies (such as mepolizumab, reslizumab and benralizumab) have all established efficacy in this patient population, have no serious safety concerns in 
clinical trials and have demonstrated favourable benefit-risk profiles [8-12, 15, 26]. In addition, IL-4 and IL-13 play a key role in the pathogenesis of asthma. Therapies that inhibit both IL-4 and IL-13 (including dupilumab) have shown some promising results [5].

All of these biologic treatments target the lower airways and associated symptoms but also have potential benefits in terms of comorbidities. These include potentially avoiding the adverse events associated with frequent oral steroid use, the rhinosinusitis and nasal polyps associated with upper airway eosinophilia, and atopic dermatitis associated with IL-4/IL-13 activity. Could the ultimate goal of anti-eosinophilic therapy be to minimise and perhaps even eliminate oral corticosteroid use, thereby consigning maintenance oral corticosteroids to history, at least in a majority of SEA patients?

There remains a need to look at how anti-eosinophilic therapies can be used to treat patients with uncontrolled disease, including children, adolescents and the elderly. In addition, patients with significant involvement of the upper airways, such as chronic rhinosinusitis with polyposis, will require a multidisciplinary team approach. Recommendations for other treatment options, including bronchial thermoplasty, macrolides and prostaglandin $\mathrm{PGD}_{2}-\mathrm{CRTH} 2$ antagonists, also need to be evaluated.

\section{Monitoring and follow-up}

When patients are prescribed a biologic therapy for SEA, a number of questions are raised. Which sort of monitoring is necessary, how should patient follow-up be conducted, and how can we determine which patients have responded to treatment? Also, under which clinical circumstances should patients stop or switch therapy [26]?

Identifying therapeutic responders and non-responders is not easy, as it may take a year or more for a reduction in clinical exacerbations to emerge. Treatment goals vary and, most of the time, a combination of clinical signals is involved, including: symptom reduction (as captured by treatment effects on the individual asthma symptom load and the Asthma Control Test (ACT) or Asthma Control Questionnaire (ACQ)); reduced exacerbation rates; restoration of the sense of smell; and overall health-status restoration. This last point includes the ability to get a good night's sleep and feeling more alert (captured by Health-Related Quality of Life (HRQoL) questionnaires such as the Asthma Quality of Life Questionnaire). However, these asthma questionnaires do not always reflect this variety in treatment goals in clinical trials involving SEA.

A number of elements contribute to response and individual responses will differ. Monitoring of lung function, symptoms, quality-of-life and exacerbation history may support early clinical decisions on treatment response. Improvements in comorbidities, rather than in asthma itself, may result in higher quality-of-life scores and data should be collected from both the upper and lower airways to evaluate treatment effect. Thus, it is important to conduct a careful characterisation of the symptom profile in order to have objective measures to follow when response is evaluated. Anti-eosinophilic therapies for SEA are not curative, so guidance on the switching and stopping of these therapies, along with other systemic (e.g. oral corticosteroids) and inhaled treatments, is important. The initial suggestion of the expert task force is to develop a traffic-light system to determine response. Similar to the management approach for omalizumab, it has been agreed that patients with SEA require treatment for at least 4 months before an initial assessment of response can be determined. Following this, patients who are intermediate responders should either continue treatment for a year to assess response, or be considered for a switch to an alternative anti-eosinophilic therapy if response is low. The initial proposal, which will require further development, is described in figure 2.

\section{Discussion and next steps}

The high clinical need in severe asthma and the introduction of effective biological therapies for severe asthma related to eosinophilia make the development of a consensus to support clinicians treating these patients an important undertaking. The ERS has expressed an interest in taking this subject forward, potentially through an ERS research seminar and through discussion in sessions at national and international congresses. The intention is to develop a position statement supported by evidence to reflect a broader expert opinion around the management of patients with severe corticosteroid-refractory asthma, and to ensure that the fast-moving scientific developments in this field are applied to clinical practice for the benefit of patients. 
FIGURE 2 The traffic-light system for response and non-response following at least 4 months of treatment with an anti-eosinophilic therapy

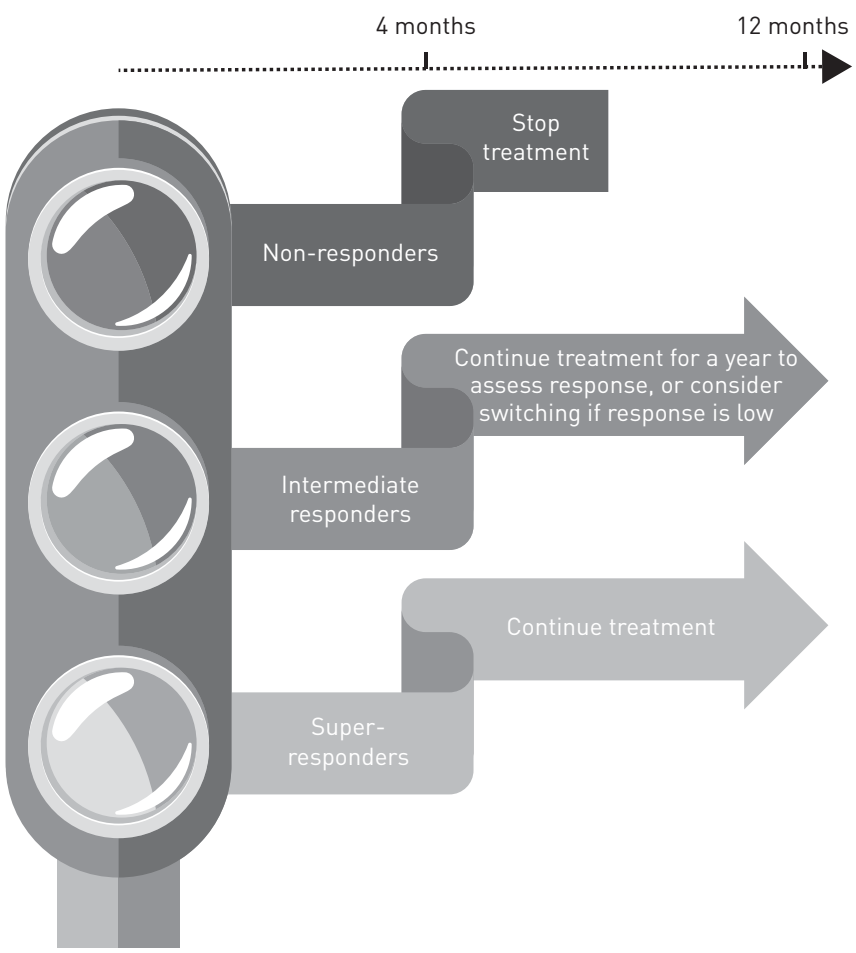

\section{Acknowledgements}

The contents of this paper reflect the discussions between attending participants at the European Consensus Meeting for Severe Eosinophilic Asthma (November 24 2016, Amsterdam, The Netherlands) where medical attendees from TEVA Pharmaceuticals Europe B.V. were present as observers. The authors have been given full editorial control and independence and TEVA has had no influence on the contents. The following members of the expert group made important contributions at the initial meeting and/or made useful comments on earlier drafts of this editorial: Ratko Djukanovic (University of Southampton, Southampton, UK), Kian Fan Chung (Imperial College, London, UK), Walter Canonica (Humanitas University, Milan, Italy), Alberto Papi (Ferrara University, Ferrara, Italy), Chris Brightling (University of Leicester, Leicester, UK), Ildiko Horváth (Semmelweis University, Budapest, Hungary), Samantha Walker (Asthma UK, London, UK), Guy Brusselle (Ghent University Hospital, Ghent, Belgium), Vibeke Backer (Bispebjerg Hospital, Copenhagen, Denmark), Piotr Kuna (University of Lodz, Lodz, Poland), Elisabeth Bel (University of Amsterdam, Amsterdam, The Netherlands), Luis Perez de Llano (Lucus Agusti University Hospital, Lugo, Spain), and Klaus Rabe (University of Kiel, Kiel, Germany).

\section{References}

1 Corren J. Asthma phenotypes and endotypes: an evolving paradigm for classification. Discov Med 2013; 15: 243-249.

2 Agusti A, Bel E, Thomas $\mathrm{M}$, et al. Treatable traits: toward precision medicine of chronic airway diseases. Eur Respir J 2016; 47: 410-419.

3 Chung KF. Targeting the interleukin pathway in the treatment of asthma. Lancet 2015; 386: 1086-1096.

4 Chung KF, Wenzel SE, Brozek JL, et al. International ERS/ATS guidelines on definition, evaluation and treatment of severe asthma. Eur Respir J 2014; 43: 343-373.

5 de Groot JC, ten Brinke A, Bel EH. Management of the patient with eosinophilic asthma: a new era begins. ERJ Open Res 2015; 1: 00024-2015.

6 Castro M, Bacharier LB. Treatment for severe eosinophilic asthma-consistent effect of anti-interleukin-5 antibodies? Lancet 2016; 388: 2059-2060.

7 Schleich FN, Chevremont A, Paulus V, et al. Importance of concomitant local and systemic eosinophilia in uncontrolled asthma. Eur Respir J 2014; 44: 97-108.

8 Bleecker ER, FitzGerald JM, Chanez P, et al. Efficacy and safety of benralizumab for patients with severe asthma uncontrolled with high-dosage inhaled corticosteroids and long-acting $\beta 2$-agonists (SIROCCO): a randomised, multicentre, placebo-controlled phase 3 trial. Lancet 2016; 388: 2115-2127.

9 Castro M, Zangrilli J, Wechsler ME, et al. Reslizumab for inadequately controlled asthma with elevated blood eosinophil counts: results from two multicentre, parallel, double-blind, randomised, placebo-controlled, phase 3 trials. Lancet Respir Med 2015; 3: 355-366.

10 Ortega HG, Yancey SW, Mayer B, et al. Severe eosinophilic asthma treated with mepolizumab stratified by baseline eosinophil thresholds: a secondary analysis of the DREAM and MENSA studies. Lancet Respir Med 2016; 4: 549-556.

11 Pavord ID, Korn S, Howarth P, et al. Mepolizumab for severe eosinophilic asthma (DREAM): a multicentre, double-blind, placebo-controlled trial. Lancet 2012; 380: 651-659. 
12 FitzGerald JM, Bleecker ER, Nair P, et al. Benralizumab, an anti-interleukin-5 receptor $\alpha$ monoclonal antibody, as add-on treatment for patients with severe, uncontrolled, eosinophilic asthma (CALIMA): a randomised, double-blind, placebo-controlled phase 3 trial. Lancet 2016; 388: 2128-2141.

13 Bel EH, Ortega HG, Pavord ID. Glucocorticoids and mepolizumab in eosinophilic asthma. N Engl J Med 2014; 371: 2433-2434.

14 Corren J, Weinstein S, Janka L, et al. Phase 3 study of reslizumab in patients with poorly controlled asthma: effects across a broad range of eosinophil counts. Chest 2016; 150: 799-810.

15 Bjermer L, Lemiere C, Maspero J, et al. Reslizumab for inadequately controlled asthma with elevated blood eosinophil levels: a randomized phase 3 study. Chest 2016; 150: 789-798.

16 Hekking PP, Wener RR, Amelink M, et al. The prevalence of severe refractory asthma. J Allergy Clin Immunol 2015; 135: 896-902.

17 de Groot JC, Storm H, Amelink M, et al. Clinical profile of patients with adult-onset eosinophilic asthma. ERJ Open Res 2016; 2: 00100-2015.

18 Haldar P, Pavord ID, Shaw DE, et al. Cluster analysis and clinical asthma phenotypes. Am J Respir Crit Care Med 2008; 178: 218-224.

19 Price DB, Rigazio A, Campbell JD, et al. Blood eosinophil count and prospective annual asthma disease burden: a UK cohort study. Lancet Respir Med 2015; 3: 849-858.

20 Arbes SJ Jr. Do all asthmatics with atopy have atopic asthma? J Allergy Clin Immunol 2012; 130: 1202-1204.

21 Wagener AH, de Nijs SB, Lutter R, et al. External validation of blood eosinophils, FE(NO) and serum periostin as surrogates for sputum eosinophils in asthma. Thorax 2015; 70: 115-120.

22 Castro M, Wenzel SE, Bleecker ER, et al. Benralizumab, an anti-interleukin 5 receptor $\alpha$ monoclonal antibody, versus placebo for uncontrolled eosinophilic asthma: a phase $2 \mathrm{~b}$ randomised dose-ranging study. Lancet Respir Med 2014; 2: 879-890.

23 Froidure A, Mouthuy J, Durham SR, et al. Asthma phenotypes and IgE responses. Eur Respir J 2016; 47: $304-319$.

24 Brandström J, Vetander M, Lilja G, et al. Individually dosed omalizumab: an effective treatment for severe peanut allergy. Clin Exp Allergy 2017; 47: 540-550.

25 Konradsen JR, Nordlund B, Nilsson OB, et al. High basophil allergen sensitivity (CD-sens) is associated with severe allergic asthma in children. Pediatr Allergy Immunol 2012; 23: 376-384.

26 Cabon Y, Molinari N, Marin G, et al. Comparison of anti-interleukin-5 therapies in patients with severe asthma: global and indirect meta-analyses of randomized placebo-controlled trials. Clin Exp Allergy 2017; 47: 129-138. 